\title{
Gastric Signet-Ring-Cell Adenocarcinoma with Delayed Retroperitoneal Metastasis and Fibrosis
}

\author{
Matthew G.K. Benesch Alexander Mathieson \\ Discipline of Surgery, Faculty of Medicine, Memorial University of Newfoundland, St. John's, \\ $\mathrm{NL}$, Canada
}

\section{Keywords}

Gastric cancer · Signet-ring cell · Adenocarcinoma $\cdot$ Retroperitoneal fibrosis

\begin{abstract}
Gastric signet-ring-cell adenocarcinoma (SRCC) is a rare disease entity, often characterized by early age of the onset and sometimes attributable to heritable genetic mutations. Overall prognosis is usually poor due to diagnosis at late stages. There are a handful of case reports that describe patient presentation with retroperitoneal fibrosis secondary to malignancy from a concurrent gastric SRCC found on the workup. No information exists on timing from primary tumor development to retroperitoneal disease. Further, there is speculation that gastric SRCC may have an indolent phase prior to symptomatic disease, but its natural history is essentially entirely unknown. In this case report, we describe a 39-year-old male with an incidentally discovered gastric SRCC who then underwent multimodality treatment with curative intent. No evidence of recurrence was documented on interval surveillance scans for 4.5 years, at which point, he rapidly developed a large retroperitoneal mass that was biopsied for metastatic disease. He succumbed to his pathology within 6 months. This presentation suggests that gastric SRCC could have both a relatively long indolent phase and an unpredictable propensity for explosive metastatic progression. Tumor biology factors that affect this balance are not understood.
\end{abstract}

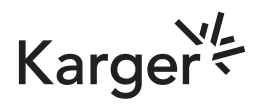




\section{Introduction}

Signet-ring-cell adenocarcinoma (SRCC) of the stomach comprises about $17 \%$ of all gastric cancer cases [1]. Despite the incidence of gastric cancer decreasing worldwide, the incidence of SRCC is rising [2]. Historically described as a "diffuse-type" gastric cancer by the Lauren classification, gastric SRCCs are now classified as poorly cohesive carcinomas composed of tumor cells with prominent cytoplasmic mucin, resulting in a crescent-shaped nucleus at the cell periphery [1,2]. Gastric SRCCs share no risk factors with conventional intestinal gastric cancers such as Helicobacter pylori infection [2]. Some gastric SRCCs arise from germline mutations in $C D H 1$, termed as hereditary diffuse gastric cancers (HDGCs); however, an increasing array of genes are now implicated including CTNNA1, BRCA2, STK11, $S B H B, P R S S 1, A T M, M S R 1$, and PALB2 [3, 4]. HDGC however comprises only 1-3\% of all gastric cancers, leaving the pathogenesis behind most gastric SRCCs unknown and understudied [5].

Prognosis of gastric SRCCs is historically poor with a median survival of 10 months [1]. If detected prior to submucosal invasion, prognosis is equivalent or better than that of intestinal gastric adenocarcinomas [2]. However, the diffuse nature of the disease usually results in detection at advanced stages $[1,4]$. There remains considerable debate from numerous retrospective epidemiological studies whether SRCC histology is an independent prognostic factor [2]. The epidemiology of metastatic patterns of gastric cancer is also poorly understood, but one Swedish study showed the most common sites are the liver (48\%), peritoneum (32\%), lung (15\%), and bone (12\%), with SRCCs more favoring the peritoneum and bone over the liver and lungs [6]. In this case report, we describe a 39-year-old male with a sudden onset of metastatic gastric SRCC to the retroperitoneum nearly 5 years after curative gastrectomy and chemoradiotherapy despite adequate surveillance. The timing and location of his highly unusual metastatic burden underscore our limited understanding of SRCC biology.

\section{Case Presentation}

An otherwise healthy 39-year-old male presented to the clinic with a 4-month history of bloating, reflux, occasional nausea, and early satiety. Proton pump inhibitor therapy provided some symptom relief. The only family history of gastrointestinal tract malignancies was two first cousins diagnosed with colon cancers in their 50-60s. An upper endoscopy exam showed moderately diffuse inflammation and edema with stigmata of bleeding in the fundus (Fig. 1). Biopsies revealed invasive adenocarcinoma, diffuse type, with signet-ring cells. Computer tomography staging of the chest, abdomen, and pelvis demonstrated no locoregional lymphadenopathy or metastatic disease, and CA 19-9 levels were negative. Given that our province has among the
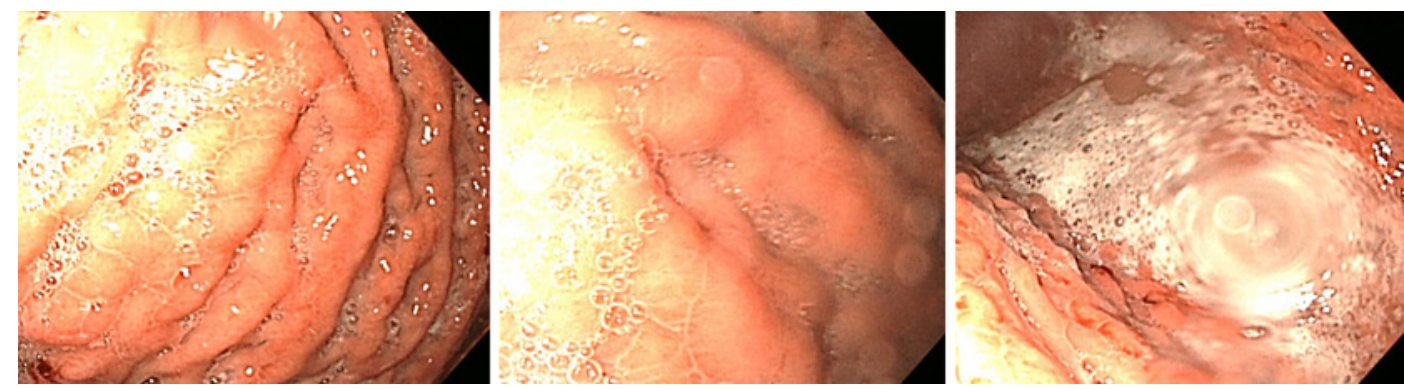

Fig. 1. Photographs of an initial upper endoscopy exam showing moderately diffuse inflammation and edema with stigmata of bleeding in the fundus of the stomach. Biopsies reveal SRCC. 
highest incidence of $C D H 1$ mutation-driven gastric SRCCs in the world [4], he underwent genetic testing for six known $C D H 1$ pathogenic variants within our patient population but was negative.

Three cycles of preoperative chemotherapy were planned. He underwent one cycle of epirubicin (50 mg/m $\mathrm{m}^{2}$ intravenous for 1 day), cisplatin $\left(60 \mathrm{mg} / \mathrm{m}^{2}\right.$ intravenous for 1 day), and capecitabine ( $625 \mathrm{mg} / \mathrm{m}^{2}$ oral twice a day for 21 days) chemotherapy, but for cycle two, cisplatin was switched to oxaliplatin $\left(130 \mathrm{mg} / \mathrm{m}^{2}\right.$ intravenous for 1 day) secondary to severe tinnitus. For the third cycle, epirubicin was reduced to $50 \%$, following issues with hepatic steatosis and elevated bilirubin, followed shortly thereafter by a 2-week hospital admission for grade 4 (life-threatening) diarrhea. Three weeks later, a total gastrectomy with D1 lymphadenectomy followed by esophagojejunostomy with roux-en-Y reconstruction with feeding jejunostomy was performed. Final pathology demonstrated ypT3N0, poorly differentiated, (grade 3) diffuse-type adenocarcinoma at the fundus/proximal body invading subserosa connective tissue, with a total of nine lymph nodes examined. There was some concern noted whether the proximal margin was microscopically involved. Given this finding and his poor tolerance with his neoadjuvant chemotherapy, he was rediscussed at multidisciplinary tumor boards. Given his previous reactions to chemotherapy, his adjuvant treatment consisted of three cycles of dose-reduced fluorouracil ( $400 \mathrm{mg} / \mathrm{m}^{2}$ intravenous for 1 day) and leucovorin (20 mg/m $\mathrm{m}^{2}$ intravenous for 1 day) with concurrent radiation (45 Gy in 25 fractions). He completed this treatment course without any severe toxicities.

One year later, this patient presented with symptoms of a small bowel obstruction, for which after workup, he was taken for a laparotomy for treatment of an internal Peterson's hernia. It was noted in this operative report that there was no evidence of metastatic disease. Apart from this, this patient completed standard of care history and physicals with surveillance CT imaging, with his scans at 4 years from surgery negative for recurrence (Fig. 2a-c).

In the following 6 months after his last surveillance scan at 4 years, this patient has issues with both renal colic requiring extracorporeal shock wave lithotripsy and prostatitis that settled with antibiotics. Concurrently, he began to complain of abdominal pain and dyspepsia with new weight loss, and hence, a repeat CT scan was arranged (4.5 years from diagnosis, 6 months from last imaging). This scan demonstrated an extensive retroperitoneal heterogenous soft-tissue mass involving the left iliopsoas extending down to the left groin with dystrophic calcification, displacing the left kidney (Fig. 2d-f). Additional magnetic resonance imaging of this mass was interpreted as nonspecific (not shown). Retroperitoneal core biopsies showed muscle infiltration by spindle cells with rare atypical epithelioid cells with large nucleoli throughout (Fig. 3a), with areas of intracellular mucin confirmed with the mucicarmine stain (Fig. 3b). This would be consistent with a signet-ring-cell morphology. These single and small clusters were also positive for cytokeratin 7 and pankeratin (Fig. 3c), with rare weak nuclear expression of caudal type homeobox 2 (Fig. 3d), supportive of a gastrointestinal epithelial source for this malignancy. HER2 protein expression by immunohistochemistry was negative (not shown). Additional SS18 gene translocation by fluorescence in situ hybridization was negative, ruling out sarcoma.

Given his worsening bowel obstructive symptoms and inability to tolerate oral intake, a laparotomy was arranged. The small bowel was found to be densely adherent to the mesentery, and a tube was ultimately placed in a loop of jejunum at a site of an inadvertent enterotomy. This patient was then palliated and passed away 5 months after his diagnosis of recurrence.

\section{Discussion and Conclusion}

To our knowledge, this is the first case presentation of retroperitoneal metastasis of gastric SRCC several years after initial diagnosis and completion of curative multimodality treatment. Multiple interval images showed no evidence of recurrent macroscopic disease for over 4 years.

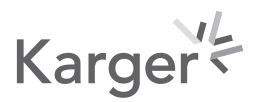



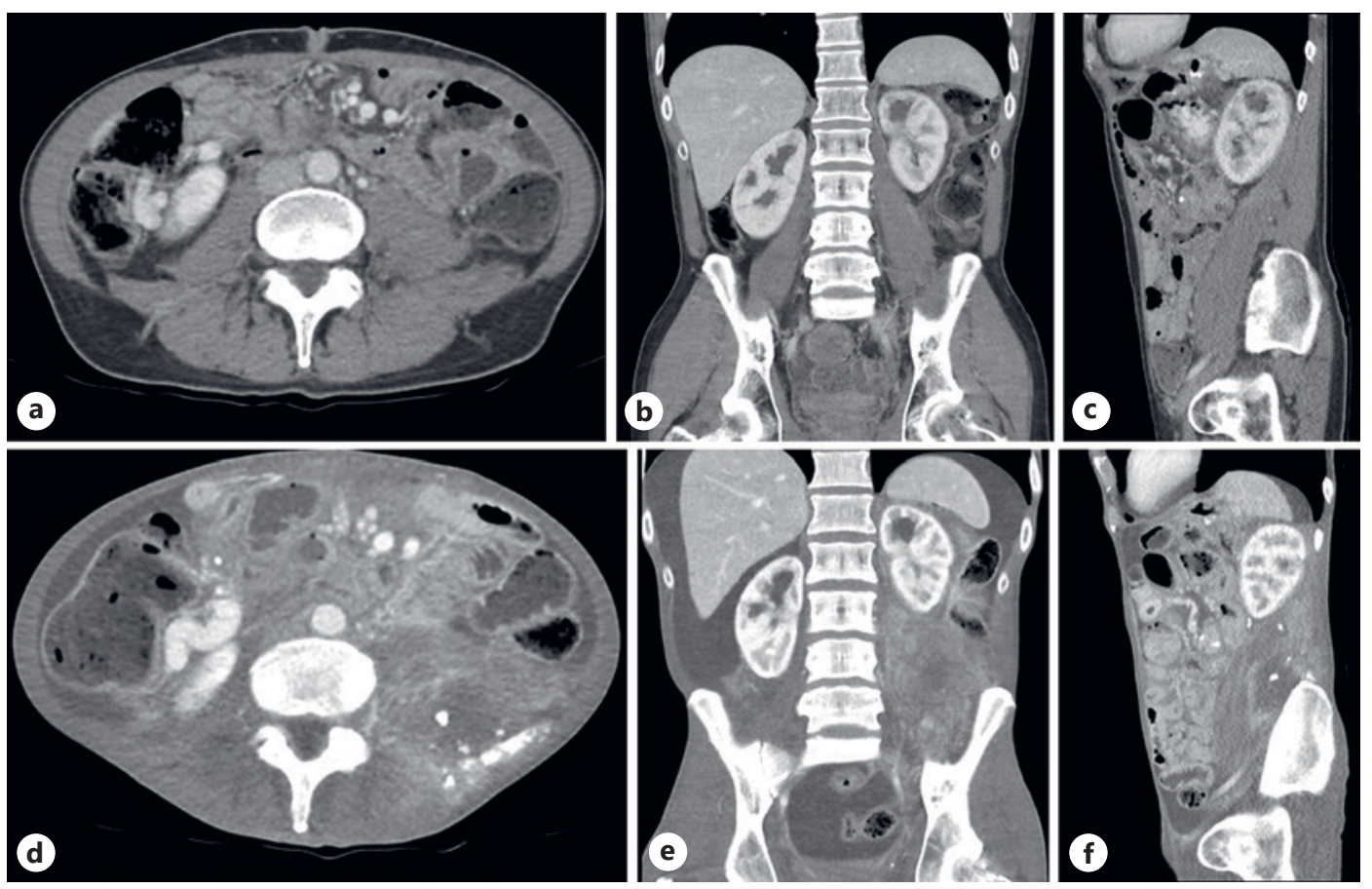

Fig. 2. Computer tomography scans demonstrating rapid development of retroperitoneal metastasis and fibrosis. Axial (a), coronal (b), and sagittal (c) views showing no evidence of disease 4 years after initial diagnosis. Axial (d), coronal (e), and sagittal (f) views showing extensive retroperitoneal disease 6 months later.

There are approximately 12 documented cases of retroperitoneal fibrosis secondary to concurrent gastric SRCC on the workup [7-9]. Typically these patients present with worsening lower back, flank, abdomen, or perineum pain secondary to localized compression [8]. Fibrotic expansion can ultimately result in ureteric obstruction and renal insufficiency [8]. Ultimately, the pathophysiology behind retroperitoneal fibrosis secondary to malignant disease is not understood, although it is likely an immune-related response secondary to an overwhelming cytokine storm that stimulates fibroblast proliferation and collagen deposition [10]. Due in part to the typically advanced stage at presentation, the performance status of these patients is often too low to tolerate palliative chemotherapy $[7,8]$.

Additionally, the long delay between diagnosis and retroperitoneal disease in this patient may provide a unique insight into gastric SRCC tumor biology. From studies of patients with CDH1 mutation-driven HDGC, endoscopic studies have demonstrated upward of $93 \%$ of asymptomatic carriers having at least $\mathrm{T} 1 \mathrm{a}$ disease [4]. This disease state is relatively indolent however the natural time course of development and progression from these mucosal cancer foci to fulminant disease is not known [4]. Among SRCCs, gastric SRCC uniquely diffusely infiltrates the gastric wall and stimulates a reactive fibrosis leading to linitis plastica. The loss of e-cadherin expression in turn mitigates the formation of large macroscopic tumors prior to distant disease dissemination [1]. Further, the response of gastric SRCC patients to conventional chemotherapy and radiotherapy regimens have not been prospectively studied [1]. One retrospective study has shown that neoadjuvant chemotherapy provides no survival benefit in patients with locally advanced gastric SRCC [11].

In synthesizing these findings and prior case reports of retroperitoneal fibrosis and gastric SRCC, we conjecture there may be a long indolent time before symptomatic disease for gastric SRCC. The 4.5-year span between diagnosis and retroperitoneal disease presentation for our patient could represent a lead time bias as his gastric primary was discovered on an 


\section{Case Reports in Oncology}

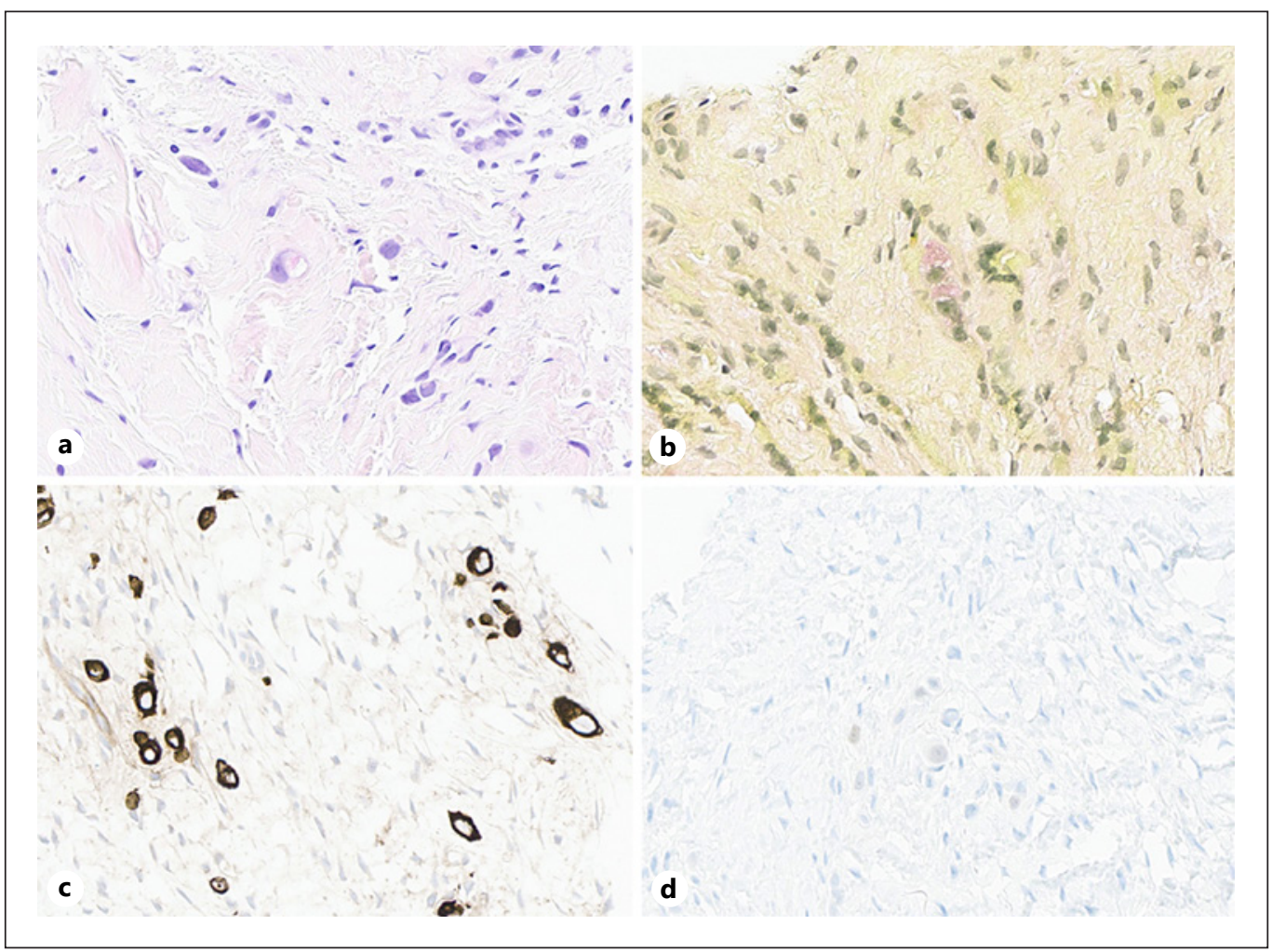

Fig. 3. Immunohistochemical staining of retroperitoneal core biopsies diagnosing metastatic carcinoma. a Hematoxylin and eosin demonstrating rare lesional cells with large vacuoles consistent with the SRCC phenotype. b Mucicarmine-positive staining suggestive of intracellular mucin consistent with signet-ringcell morphology. Pankeratin- (c) and CDX2- (d) positive staining indicative of an epithelial origin. CDX2, caudal type homeobox 2 .

incidental gastroscopy. Despite standard of care surveillance and maximal neo- and postadjuvant treatment, the explosive development of retroperitoneal disease underscores our poor understanding of gastric SRCC tumor biology. Clinicians managing these patients need to be carefully wary of otherwise vague or seemingly unconnected abdominal and perineal symptomology in order to effectively provide palliative surgical options to minimize obstructive complications in patients with retroperitoneal fibrosis secondary to malignancy.

\section{Acknowledgments}

We thank the family of the patient presented in this case report for allowing us to share his story to improve the understanding of gastric SRCC tumor biology. We thank Dr. Angela Tate for providing photographs of immunohistochemical slides.

\section{Statement of Ethics}

This retrospective review of patient data did not require ethical approval in accordance with local/national guidelines. Written informed consent has been obtained from the spouse of this deceased patient for publication of this case report and accompanying images. 


\section{Conflict of Interest Statement}

The authors have no conflicts of interest to declare.

\section{Funding Sources}

The authors received no financial support for the authorship.

\section{Author Contributions}

Matthew Benesch drafted the manuscript. Alex Mathieson provided clinical information and directed patient care. All the authors participated in manuscript revision and approved the final version.

\section{Data Availability Statement}

All data generated or analyzed during this study are included in this article. Further inquiries can be directed to the corresponding author.

\section{References}

1 Benesch MGK, Mathieson A. Epidemiology of signet ring cell adenocarcinomas. Cancers. 2020 Jun 11;12(6): 1544.

2 Pernot S, Voron T, Perkins G, Lagorce-Pages C, Berger A, Taieb J. Signet-ring cell carcinoma of the stomach: impact on prognosis and specific therapeutic challenge. World J Gastroenterol. 2015;21(40):11428-38.

3 Hansford S, Kaurah P, Li-Chang H, Woo M, Senz J, Pinheiro H, et al. Hereditary diffuse gastric cancer syndrome: CDH1 mutations and beyond. JAMA Oncol. 2015 Apr;1(1):23-32.

4 Benesch MGK, Bursey SR, O'Connell AC, Ryan MG, Howard CL, Stockley CC, et al. CDH1 gene mutation hereditary diffuse gastric cancer outcomes: analysis of a large cohort, systematic review of endoscopic surveillance, and secondary cancer risk postulation. Cancers. 2021 May 26;13(11):2622.

5 Kaurah P, Talhouk A, MacMillan A, Lewis I, Chelcun-Schreiber K, Yoon SS, et al. Hereditary diffuse gastric cancer: cancer risk and the personal cost of preventive surgery. Fam Cancer. 2019 0ct;18(4):429-38.

6 Riihimäki M, Hemminki A, Sundquist K, Sundquist J, Hemminki K. Metastatic spread in patients with gastric cancer. Oncotarget. 2016;7(32):52307-16.

7 Karbasi A, Karbasi-Afshar R, Ahmadi J, Saburi A. Retroperitoneal fibrosis as a result of signet ring cell gastric cancer: a case-based review. J Gastrointest Cancer. 2013 Mar;44(1):94-7.

8 Peixoto RD, Al-Barrak J, Lim H, Renouf D. Gastroesophageal cancer and retroperitoneal fibrosis: two case reports and review of the literature. World J Gastrointest Oncol. 2013 Mar 15;5(3):68-70.

9 Shetty NS, Calhoun A, Sunjaya D, Greer A, Willingham FF. Retroperitoneal fibrosis with skeletal muscle invasion as an early manifestation of metastatic gastric cancer. ACG Case Rep J. 2021 Apr;8(4):e00553.

10 Vaglio A, Salvarani C, Buzio C. Retroperitoneal fibrosis. Lancet. 2006 Jan 21;367(9506):241-51.

11 Li Y, Ma FH, Xue LY, Tian YT. Neoadjuvant chemotherapy vs upfront surgery for gastric signet ring cell carcinoma: a retrospective, propensity score-matched study. World J Gastroenterol. 2020 Feb 28;26(8):818-27.

\section{Karger'}

